\title{
Variations in weight stigma concerns
}

\author{
Jessica E. Cornick, ${ }^{1}$ Cambridge Teter, ${ }^{1}$ Andrew K.Thaw ${ }^{2}$ \\ ${ }^{1}$ University of California, Santa Barbara, CA; ${ }^{2}$ Millsaps College, Jackson, MS, USA
}

\section{Abstract}

Over the past 40 years, obesity rates in the United States have grown significantly; these rates have not grown uniformly across the United States (18 of the 20 counties with the highest obesity rates are located in the South). Obesity increases cardiovascular disease risk factors and new research has highlighted the negative psychological effects of obesity, known as weight stigma, including decreased selfcontrol resources, over eating, and exercise avoidance. The primary objective of this study was to determine if weight stigma concerns varied regionally and if social behaviors influenced this variation. In two studies, we collected cross-sectional data from participants in the United States including height and weight, weight stigma concerns, and perception of friends' preoccupation with weight and dieting. We also collected each participant's home zip code which was used to locate local obesity rate. We established differences in the relationship between body mass index and weight stigma concerns by local county obesity rate and showed that perceived friend preoccupation with weight and dieting mediated this relationship for individuals in low and medium obesity rate counties. For individuals living in United States counties with lower levels of obesity, increases in personal body mass index leads to increased weight stigma concerns due to an increase in perceived friend preoccupation with weight and dieting. These results indicate that relationships between body mass index, weight stigma concerns, and social networks vary significantly for subpopulations throughout the United States.

\footnotetext{
Correspondence: Jessica Cornick, Department of Psychology, University of California, Santa Barbara, CA 93106, USA.

Tel.: + 1.805.893.5798.

E-mail: cornick@psych.ucsb.edu
}

Key words: Weight stigma concerns, Obesity, BMI.

Contributions: the authors contributed equally.

Conflict of interest: the authors declare no potential conflict of interest.

Received for publication: 17 May 2016.

Revision received: 26 September 2016.

Accepted for publication: 3 October 2016.

This work is licensed under a Creative Commons AttributionNonCommercial 4.0 International License (CC BY-NC 4.0).

(O) Copyright J.E. Cornick et al., 2016

Licensee PAGEPress, Italy

Health Psychology Research 2016; 4:6003

doi:10.4081/hpr.2016.6003

\section{Introduction}

Over the past 40 years, adult obesity rates in the United States have reached an alarming level of $33.8 \%$ (68\% are overweight OR obese; Finkelstein et al., 2012; Ogden, Carroll, Kit and Flegal, 2012). Importantly, obesity rates are not uniformly distributed across the nation; 18 of the 20 counties ranked highest for obesity are located in the South and 23 of the 25 states with the highest obesity rates are in the South and Midwest (Dwyer-Lindgren et al., 2013; The State of Obesity, 2014). While the negative physical consequences of obesity (e.g., cardiovascular diseases) have been well documented (cf. Lavie, McAuley, Church, Milani, and Blair, 2014), new research highlights harmful psychological risk factors associated with obesity (Puhl and Heuer, 2010; Pearl and Puhl, 2016). Overweight and obese individuals face pervasive stigmatization in many domains including peer networks, employment, education, healthcare, and portrayals in the media as a result of the widespread negative societal bias against overweight and obese individuals (Fikkan and Rothblum, 2012; Pearl and Puhl, 2016). It has been suggested that obese individuals are as highly stigmatized as other targets of stigma including those with AIDS, drug addiction, and criminal behavior (Sobal, 2004). The social experience of weight based stigmatization has been posited to contribute to the negative impact of weight on physical health above and beyond the physical consequences of obesity and has lasting effects on the individual's health and well-being (Hunger and Major, 2015).

Repeated exposure to weight based stigmatization increases weight bias internalization and concerns about experiencing weight based stigmatization in the future, which can undermine health in overweight individuals (Major, Eliezer, and Rieck, 2012; Pearl and Puhl, 2016). Weight stigma concerns (WSC) increase with body mass index (BMI) and perceived levels of discrimination, and explain the relationship between BMI and self-reported health, such that individuals with higher WSC have poorer self-reported health (Hunger and Major, 2015). Weight stigma concerns also explicate the relationship between past instances of discrimination and psychological and physical health with increased perceptions of discrimination relating to increased stigma concerns, which negatively predict psychological and physical health (Hunger and Major, 2015). This relationship between WSC and poorer health outcomes is attributed to a host of negative downstream consequences such as reduced self-control, resulting in over eating and exercise avoidance (reinforcing the obesity cycle; Major, Hunger, Bunyan and Miller, 2015; Tomiyama, 2014; Vartanian and Novak, 2011). While these effects have been studied mainly in overweight and obese populations, there is a pervasive societal stigma that "fat is bad" which results in non-obese individuals also being concerned about becoming overweight (Rothblum, 1992). Clearly, social norms and biases against obesity (and subsequent stigmatization and discrimination) in an individual's social network can affect an individual's health and well-being.

Social influences on health behaviors (such as smoking and alcohol use) have been well documented (Christakis and Fowler, 2008; Piko, Luszczynska, Gibbons, and Teközel, 2005; Rosenquist, Murabito, Fowler, 
and Christakis, 2011) and new research highlights the interaction of social experiences and genetic causes to influence obesity rates (Albuquerque, Stice, Rodríguez-López, and Nóbrega, 2015). For example, overweight and obese individuals are more likely to have romantic partners, friends, and family members who are overweight or obese; an individual's chance of becoming obese increases $57 \%$ if a friend becomes obese (40\% for a sibling, and $37 \%$ for a spouse; Christakis and Fowler, 2007; Leahey, LaRose, Fava, and Wing, 2011). Similarly, individuals' intentions to lose weight (and their actual success) are increased when they have more peers that are trying to lose weight (Leahey et al., 2011; Leahey, Kumar, Weinberg, and Wing, 2012). This has been explained by an increased frequency of discussions about social norms for weight loss and eating behavior (Leahey et al., 2012; Robinson, Thomas, Aveyard, and Higgs, 2014). Although these social experiences can have positive health outcomes for the individual, they could have unintended negative psychological consequences such as preoccupation with eating, weight, and obesity. Previous research has established that BMI varies by individual, local obesity rate varies regionally, and that there is a direct positive relationship between BMI and WSC (Hunger and Major, 2015; Hunger, Major, Blodorn, and Miller, 2015). Additionally, research has shown that while peers can influence health behavior, overweight and obese individuals express similar weight stigmatization levels as their non-overweight peers (Latner, Stunkard, and Wilson, 2005). However, evidence of potential regional differences in WSC, as a function of both varying local obesity rates and social experiences, is lacking. While most individuals in the US are aware of weight stigma, perhaps living in a community with higher obesity rates could insulate overweight and obese individuals from the effects of weight based stigma. Also, perhaps having a social network that does not focus on weight and eating could reduce WSC for individuals with high BMI. Both outcomes could be due to the shifting standards for obesity in the United States as there has been a significant decline in the probability of self-classifying as overweight in the last two decades. This suggests that new standards for overweight and obese are being generated in communities around the country as people avoid being labeled as overweight, which could provide a protective factor (cf. Burke, Heiland, and Nadler, 2010). The current exploratory research examined regional differences in the relationship between BMI and WSC. Based on findings from previous research, in study 1 we tested the hypothesis that the relationship between BMI and WSC was moderated by local levels of obesity. In study 2, we tested whether the relationship between BMI and WSC was mediated by perceived friend preoccupation with weight and dieting and if this overall mediation was moderated by county obesity rate (study 2 ). While the nature of this research is exploratory, we hypothesized that the relationship between BMI and WSC would be stronger in regions with lower rates of obesity as there may be more social experiences in which social pressure to avoid obesity is promoted (study 1). Additionally, the relationship between BMI and WSC would be mediated by friend preoccupation for those individuals living in lower obesity counties (study 2). Specifically, we predicted this relationship because higher BMI individuals with social networks comprised of fewer obese individuals may experience pressure, both implicit and explicit, from their social networks to conform to a normal weight based on pervasive societal standards for weight (Rothblum, 1992).

\section{Study 1}

\section{Participants}

Participants from the undergraduate subject pool at a small college in Mississippi and a university in California completed the survey in exchange for partial course credit in Introduction to Psychology and Introduction to Research Methods courses.

\section{Low obesity rate county}

The low obesity rate county consisted of a sample from a California county (22.5\% obesity rate; Centers for Disease Control, 2015) of 131 participants ( 97 female; $M$ age $=18.94, S D=1.71$ ) with an average BMI of $22.52(S D=3.32$, range $=15.83-37.87)$. Based on Centers for Disease Control (CDC; 2014) guidelines, 6\% $(\mathrm{n}=8)$ were underweight, $80 \%$ $(\mathrm{n}=105)$ were normal weight, $10 \%(\mathrm{n}=13)$ were overweight, and $4 \%$ $(\mathrm{n}=5)$ were obese. The sample was $34.4 \%$ White, $1.5 \%$ African American, 23.7\% Asian, 27.5\% Hispanic, and 12.2\% Mixed or Other ethnicity.

\section{High obesity rate county}

The high obesity rate county consisted of a sample from a Mississippi county (32.3\% obesity rate; Centers for Disease Control, 2015 ) of 81 participants (44 female; $M$ age $=20.30, S D=4.61$ ) with an average BMI of $24.65(S D=4.21$, range $=15.70-37.40)$. Based on CDC (2014) guidelines, $2.4 \%(n=2)$ were underweight, $48.1 \%(n=39)$ were normal weight, $37.1 \%(\mathrm{n}=30)$ were overweight, and $12.4 \%(\mathrm{n}=10)$ were obese. The sample was $69.1 \%$ White, $17.3 \%$ African American, $3.7 \%$ Asian, 6.2\% Hispanic, and 3.7\% Mixed or Other ethnicity.

\section{Measures}

\section{Body mass index}

Participants were asked to report their weight (in pounds) and height (in inches) for calculation of BMI $(M=23.10, S D=3.85)$. The conventional BMI formula was used: BMI = [weight / (height* height)] $\times 703$.

\section{Weight stigma concerns scale}

Participants responded to five statements (e.g. I am concerned that other people's opinion of me will be based on my weight) on a scale from 1 (strongly disagree) to 7 (strongly agree; Hunger and Major, 2015). The measure showed high internal reliability $(\alpha=0.92)$ with an average of $3.12(S D=1.44)$.

\section{Design and procedure}

The quasi-experimental study examined outcomes from a sample from a Western US county with a low obesity rate and a sample from a Southern US county with a high obesity rate. Participants arrived at the laboratory in their respective locations, provided consent and were seated at a computer terminal where the survey was accessed via the website Qualtrics. Participants were informed that participation was voluntary, were able to skip questions with no penalty, and could withdraw from the survey at any time. Upon completion, participants were fully debriefed and received research credit. All procedures were approved by the Human Subjects Committees at the college and university where data was collected.

\section{Results and Discussion}

The sample was screened for missing values and normality and subsequently the interaction of BMI and local county obesity rate on WSC was modeled (Figure 1). We hypothesized that BMI would be more strongly associated with WSC in the low obesity rate county than in the high obesity rate county as concern for weight based stigmatization may be higher in the former. To begin, we tested whether there were BMI differences by location: participants in the low obesity rate county $(M=22.19, S D=3.31)$ had a lower average BMI than those in the high 
obesity rate county $(M=24.58, S D=4.20), t(140.50)=-4.36, \mathrm{P}<0.001$. Also, consistent with previous research (Fikkan and Rothblum, 2012), a main effect of gender on WSC was found; women $(M=3.20, S D=0.13)$ had higher WSC than men $(M=2.76, S D=0.17), F(1,208)=4.21, \mathrm{P}<0.05$.

To test our hypothesis that local obesity rate moderated the relationship between BMI and WSC, we used the SPSS PROCESS macro (Hayes, 2012). Testing the moderation of the relationship between BMI and WSC by local obesity rate, there were significant main effects of both BMI $(b=0.08, t(208)=2.94,95 \% \mathrm{CI}[0.03,0.13], \mathrm{P}<0.01)$ and local county obesity rate $(b=-0.80, t(208)=-3.83,95 \%$ CI $[-1.20,-0.38]$, $\mathrm{P}<0.001)$ on WSC and a trending interaction $(b=-0.08, t(208)=-1.41$, $95 \%$ CI $[-0.18,0.03], \mathrm{P}=0.12$ ), explaining $8.7 \%$ of the variance in WSC, $F(3,207)=6.78, \mathrm{P}<0.001$ (Figure 2). Participants with higher BMI and those in the low obesity rate county reported higher WSC than participants in the high obesity rate county and those with lower BMI. Although the interaction between BMI and local county obesity rate was non-significant, we explored the simple effects of BMI for both locations and discovered that the relationship between BMI and WSC was significant for low obesity rate county participants (simple $b=0.11$, $t(208)=2.90,95 \%$ CI $[0.03,0.18], \mathrm{P}<0.01)$ but not for the high obesity rate county participants (simple $b=0.03, t(208)=0.90,95 \% \mathrm{CI}[-0.04$, 0.11 ], $\mathrm{P}=0.37$ ). As a low obesity rate county participant's BMI increased, WSC increased. Additionally, participants with low BMI had similar levels of WSC (simple $b=-0.51, t(208)=-1.70,95 \%$ CI $[-1.10$, 0.08 ], $\mathrm{P}=0.09$ ) but participants with high $\mathrm{BMI}$ in the low obesity rate county had significantly higher levels of WSC relative to participants with high BMI in the high obesity rate county (simple $b=1.07, t(208)=-$ $3.87,95 \%$ CI [-1.62, -0.53$], \mathrm{P}<0.001$ ). Study 1 provided support (through a trending interaction) that local county obesity rate moderates the relationships between BMI and WSC such that increased BMI was related to higher WSC (with individuals in the low obesity county having significantly higher WSC levels compared to individuals in the high obesity rate county). However, only in the low obesity county were increasing BMI values associated with increasing levels of WSC. These findings are the first to demonstrate a regional difference in WSC and also suggest that WSC is not dependent solely on physical size, but instead may be related to weight based norms in peer groups and the community. Study 2 examined whether perceived friend preoccupation with weight and dieting mediated the moderated relationship of local obesity rate, BMI and WSC in a national sample of US adults (Figure 3).

\section{Study 2}

\section{Participants}

Participants were recruited from Amazon's Mechanical Turk (MTurk, $\mathrm{n}=180,110$ female; $M$ age $=36.68, S D=13.60$ ) with an average BMI of $27.18(S D=6.54$, range=15.66 -56.64). Based on CDC (2014) guidelines, $4 \%(n=7)$ were underweight, $40 \%(n=71)$ were normal weight, $29 \%(\mathrm{n}=52)$ were overweight, and $27 \%(\mathrm{n}=48)$ were obese. The sample was $80.6 \%$ White, 3.9\% African American, 6.1\% Asian, 3.3\% Hispanic, and $5.6 \%$ Mixed or Other ethnicity.

\section{Measures}

\section{Body mass index}

Participants were asked to report their weight (in pounds) and height (in inches) for calculation of BMI $(M=27.51, S D=7.02)$. The conventional BMI formula was used BMI = [weight / (height* height) $]$ $\times 703$.
Weight stigma concerns scale

As in study 1, participants responded to five statements (e.g. I am concerned that other people's opinion of me will be based on my weigh') on a scale from 1 (strongly disagree) to 7 (strongly agree; Hunger and Major, 2015). The measure showed high internal reliability $(\alpha=0.96)$ with an average of $3.61(S D=1.74$.

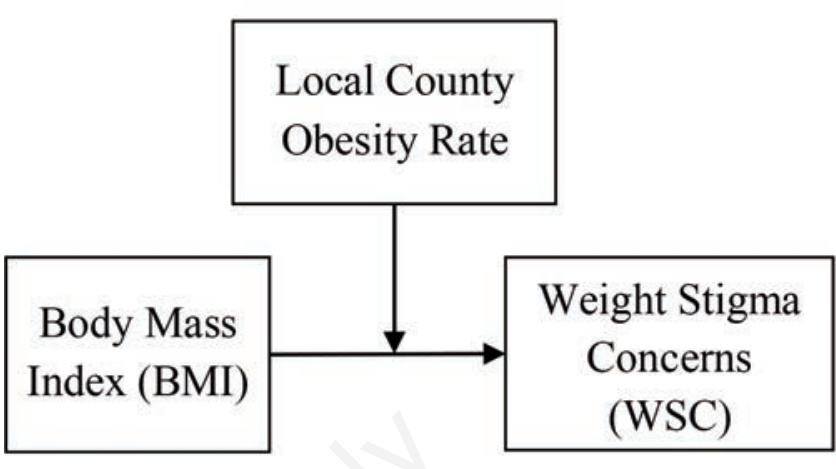

Figure 1. Study 1 moderation model.

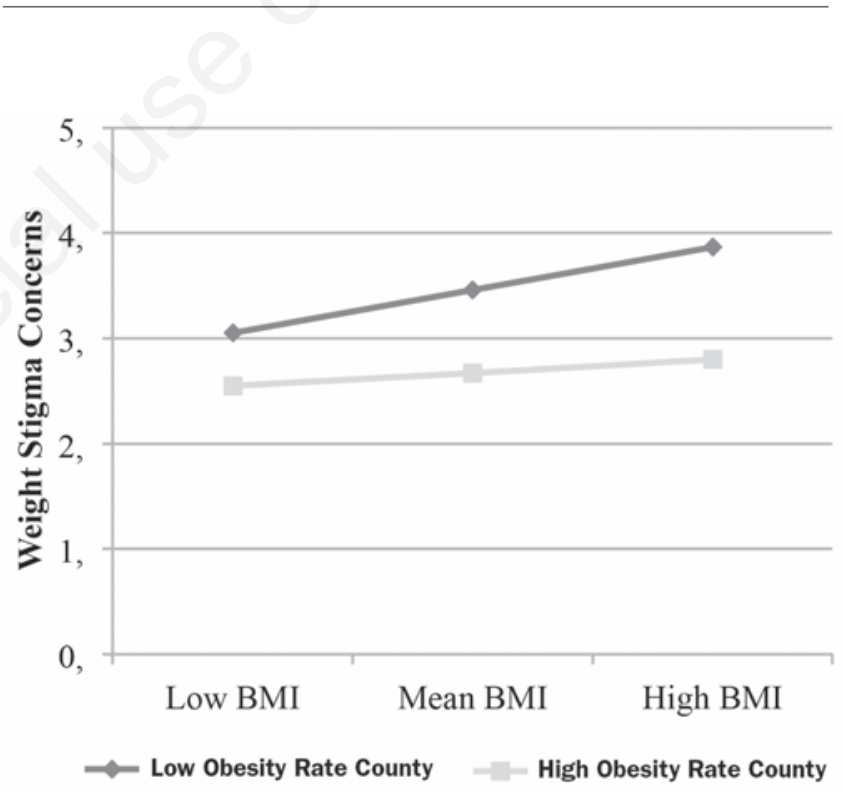

Figure 2. Association between body mass index (BMI) and weight stigma concerns (WSC) for participants in California and Mississippi at \pm 1 standard deviation from the mean of BMI (BMI, $\mathrm{M}=23.10, \mathrm{SD}=3.85$ ).

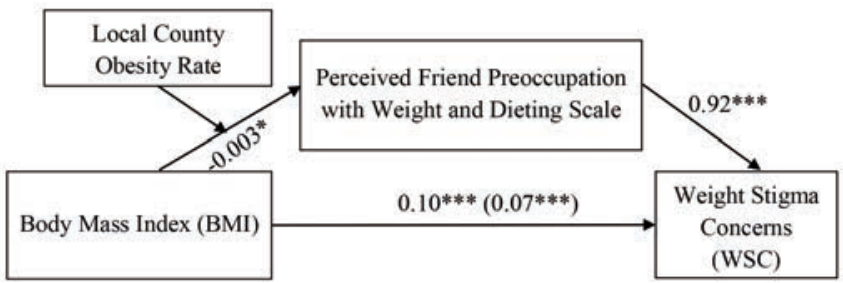

Figure 3. PROCESS results for moderated mediation model in Study 2. Unstandardized coefficients (b) are presented. ${ }^{*} \mathrm{P}<0.05$, ${ }^{* *} \mathbf{P}<0.01,{ }^{* * *} \mathbf{P}<0.001$. 


\section{Local county obesity rate}

Participants were asked to provide their home zip code at the end of the study which was used to locate their home county and state information. Then using the CDC (2015) community health status indicators website, we retrieved the home county adult obesity rate for each participant.

\section{Perceived friend preoccupation with weight and dieting scale}

Participants indicated frequency of diet and eating related conversations among friends with nine statements (e.g., How often do your friends comment on each other's weight, How often do your friends worry about what they eat, How often do your friends diet) on a scale from 1 (Never) to 5 (Always; Schutz, Paxton, and Wertheim, 2002). The measure showed high internal reliability $(\alpha=0.89)$ with an average of $2.64(S D=0.78)$.

\section{Design and Procedure}

The correlational study examined outcomes from a national sample of adults on Amazon's MTurk. Participants chose to participate in the study by choosing it from a list of jobs on MTurk which linked them with the survey hosted on the website Qualtrics. Participants were informed that participation was voluntary, were able to skip questions with no penalty, and could withdraw from the survey at any time. Upon completion, participants were fully debriefed and received research payment. All procedures were approved by the Human Subjects Committees at the university where data was collected.

\section{Results and Discussion}

The sample was screened for missing values and normality; descriptive statistics and correlations were assessed. As in study 1, women reported higher levels of WSC than men [Women $(M=3.73, S D=1.79)$, Men $(M=3.20, S D=1.63), \mathrm{t}(178)=-2.01, \mathrm{P}<0.05]$ and perceived friend preoccupation with weight and dieting [Women $(M=2.77, S D=0.75)$, Men $(M=2.43, S D=0.79), t(178)=-2.94, \mathrm{P}<0.01$; Fikkan and Rothblum, 2012]. While BMI was not significantly different by gender [Women $(M=27.24, S D=7.40)$, Men $(M=27.09, S D=4.95), t(176)=-0.15, \mathrm{P}=0.88)$, interestingly, women in our sample lived in counties with higher adult obesity rates [Women $(M=27.30, S D=5.50)$, Men $(M=25.97, S D=4.41)$, $t(175)=-1.70, \mathrm{P}=0.09]$

We assessed correlations amongst our variables of interest (Table 1). County level obesity rate was positively correlated with BMI but not WSC; perceived friend preoccupation with weight and dieting was positively correlated with BMI and WSC, and BMI and WSC were positively correlated. Of central importance to this paper, local county obesity rate and perceived friend preoccupation with weight and dieting were positively correlated providing additional support for perceptions of friend preoccupations underpinning the relationship of BMI and WSC.

The moderation by local county obesity percentage of the mediation of BMI and WSC by perceived friend preoccupation with weight and dieting was modeled using the SPSS PROCESS macro (Hayes, 2012; Figure 2). Ten thousand bootstrap samples were used to create $95 \%$ bias-correct and accelerated confidence intervals. We hypothesized that the relationship between BMI and WSC would be mediated by friend preoccupation for those individuals living in lower obesity counties. Results revealed a significant total effect between BMI and WSC $(b=0.10, \mathrm{P}<0.001)$, which remained significant once perceived friend preoccupation with weight and dieting was included in the model ( $b=0.07, \mathrm{P}<0.001$; see Figure 3 ). Both BMI and local county obesity rate significantly predicted perceived friend preoccupation with weight and dieting [BMI $(b=0.02,95 \% \mathrm{CI}[0.01,0.03], \mathrm{P}<0.05)$, obesity rate $(b=0.04,95 \%$ CI $[0.01,0.06], \mathrm{P}<001)]$, however these main effects were qualified by a significant interaction $(b=-.003,95 \% \mathrm{CI}[-0.004,-$
0.003], P<0.05; Figure 4). Participants with higher BMI and those that lived in counties with higher obesity rates reported higher perceived friend preoccupation with weight and dieting compared to individuals with lower BMI and those living in counties with lower obesity percentages. We then explored the conditional indirect effects; the mediation of relationship between BMI and WSC by friend preoccupation was significant for those at low county obesity (simple $b=0.03,95 \% \mathrm{CI}[0.01$, $0.05], \mathrm{P}<0.01$ ) and for those at medium county obesity (simple $b=0.02$, $95 \%$ CI $[0.003,0.03], \mathrm{P}<0.01)$, but not for those at high county obesity (simple $b=0.01,95 \% \mathrm{CI}[-0.01,0.02], \mathrm{P}=0.48$; Figure 4). Additionally, participants with low BMI had significantly different levels of perceived friend preoccupation (simple $b=0.03,95 \% \mathrm{CI}[0.01,0.05], \mathrm{P}<0.01$ ), with low BMI individuals living in low obesity counties perceiving the least preoccupation relative to low BMI individuals living in high obesity counties (Figure 4); there was no significant difference by county obesity rate for those with high BMI (simple $b=0.003,95 \%$ CI [-0.01, 0.02], $\mathrm{P}=0.66$ ). Finally, participants who perceived higher friend preoccupation with weight and dieting had higher WSC $(b=0.92,95 \%$ CI $[0.68$, 1.17], $\mathrm{P}<0.001)$.

The final test was to determine if the mediation of the relationship between BMI and WSC by perceived friend preoccupation with weight and dieting was moderated by local county obesity rate via a bootstrap confidence interval for the index of moderated mediation (Hayes, 2015). The mediation of BMI and WSC by perceived friend preoccupation with weight and dieting was significantly moderated by local county obesity rate (index $=-0.002,95 \% \mathrm{CI}[-0.005,-0.0004]$ ). For individuals in low and medium obesity rate counties, the relationship between BMI and WSC was significantly mediated by perceived friend preoccupation with weight and dieting while no significant mediation existed for those in counties with high obesity rates (Figure 4).

Study 2 extended the results of study 1 by showing that local county obesity rate was a significant moderator of the BMI and WSC relationship and by demonstrating that perceived friend preoccupation with weight and dieting is one method in which increases in BMI result in increases in WSC. Study 2 also demonstrated that individuals with high BMI and those living in high obesity rate counties are most likely to perceive high levels of friend preoccupation with weight and dieting.

\section{Discussion and Conclusions}

Across two exploratory studies, we found that local county obesity rates moderated the relationship between BMI and WSC and that perceived friend preoccupation with weight and dieting partially explained the relationship between BMI and WSC. These findings add to research that demonstrates perceived discrimination increases WSC via social norms (such as perceived friend preoccupation with weight and dieting) communicated through social experiences (Hunger and Major,

Table 1. Summary of correlations, means, and standard deviations among variables in Study 2.

\begin{tabular}{lcccc} 
Measure & BMI & WSC & PFPWD & Obesity \\
BMI & - & & & \\
WSC & $.39^{* * *}$ & - & & \\
\hline PFPWD & $0.16^{*}$ & $0.41^{* * *}$ & - & \\
Obesity & $0.27^{* * *}$ & 0.05 & $0.23^{* *}$ & - \\
\hline M & 27.18 & 3.61 & 2.64 & 26.78 \\
SD & 6.54 & 1.74 & 0.78 & 5.13 \\
\hline
\end{tabular}

Weight stigma concerns (WSC), body mass index (BMI), perceived friend preoccupation with weight and dieting (PFPWD), and local obesity county rate (obesity). ${ }^{*} \mathrm{P}<0.05,{ }^{* *} \mathrm{P}<0.01,{ }^{* * *} \mathrm{P}<0.001$. 
2015). Specifically, we showed in study 1 that participants with higher BMI and those in the low obesity rate county reported higher WSC and the relationship between BMI and WSC was stronger for individuals in the low obesity rate county. To further evaluate this relationship, we assessed whether social experiences such as friend preoccupation with weight and dieting influenced the relationship between BMI and WSC. In study 2, we showed that individuals with high BMI and those living in high obesity rate counties were most likely to perceive high levels of friend preoccupation with weight and dieting and therefore, increased levels of WSC. This can leave these individuals particularly vulnerable to the negative psychological and physical risk factors associated with WSC such as decreased executive resources, increased instances of anxiety and depression, and poorer health (Hatzenbuehler, Keyes, and Hasin, 2009; Hunger and Major, 2015; Major et al., 2012).

The current work adds to research on WSC and obesity by providing the first evidence that WSC varies regionally. While prior work has shown that psychologically close-others influence weight outcomes (more than geographically close-others), our findings underscore that geographically close-others influence the types of conversations peers have (dieting and weight based) and in turn, WSC (Christiakis and Fowler, 2007; Leahey et al., 2011). This outcome is of particular concern for individuals with a high BMI and individuals living in high obesity rate counties for these individuals perceive higher levels of friend preoccupation with weight and dieting, leading to higher WSC and related negative long term outcomes (Hunger and Major, 2015).

Although this unique work represents an important first step toward understanding regional differences in WSC, there are limitations that must be addressed. First, variables were collected in a cross sectional manner and therefore causal claims should be assessed cautiously. Future studies could experimentally manipulate WSC or peer norms about weight, dieting, or eating to better document causal mechanisms in the relationships outlined in this paper. Second, the influence of socioeconomic status (SES) was not included in the model; while we do not believe this detrimentally influenced the current work (in study 1, both schools report similar average income across students and in study 2 income [Income was gathered using county level average income from the U.S. Census Bureau] was not significantly correlated

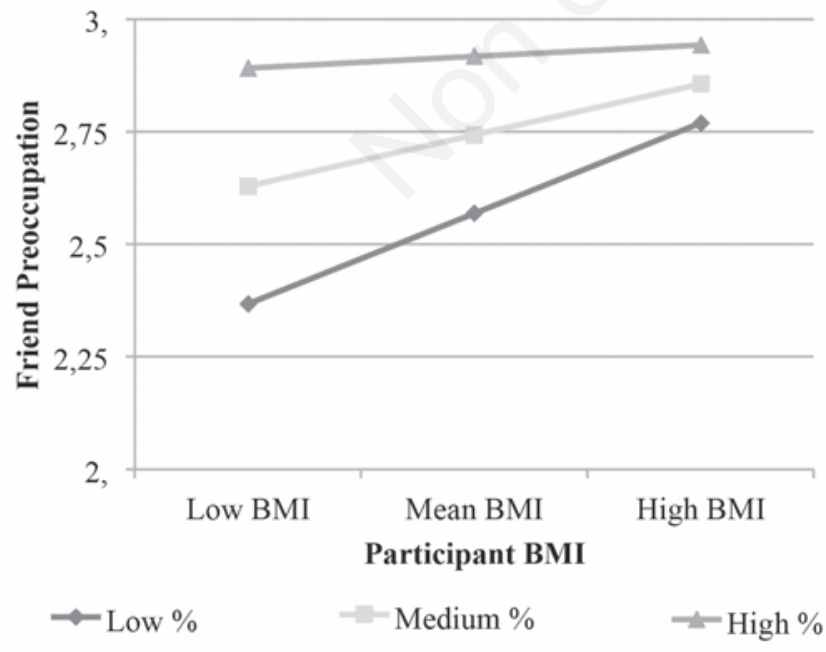

Figure 4. Association between body mass index (BMI; at \pm 1 standard deviation from the mean; $M=27.51, S D=7.02$ ) and Perceived Friend Preoccupation with Weight and Dieting for participants in low, medium, and high obesity rate counties (at \pm 1 standard deviation from the mean; county obesity rate, $M=26.78$, $\mathrm{SD}=5.13)$. with BMI or WSC), SES has been shown to influence health outcomes and should be included in future studies (Ulijaszek, 2012).

Next, the samples were predominately White which limits the generalizability of the findings as there is considerable evidence that weight and associated weight bias varies by ethnicity (Bennett and Wolin, 2006; Rand and Kuldau, 1990; Wells, 2012). White individuals are more likely to perceive themselves as overweight as compared to Black and Hispanic individuals. In the current work, BMI and WSC were positively correlated but participants were not asked to directly categorize themselves as under, normal, or over weight, thereby limiting the current work's direct application to previous research. In future studies, the moderating role of race in the relationship among BMI, WSC, and social behavior should be assessed so as to better understand its influence on these variables.

Finally, in study 1 , sample sizes in the low and high obesity rate counties were not equal. As this research was exploratory and the first of its kind to assess differences in WSC regionally, collecting maximum amounts of data from the available samples was the goal of the current work. Now that evidence of the variation in the levels of WSC has been documented and effect sizes established, power analyses can be used to calculate appropriate cell sizes for future samples. Although this research did not have an established effect size, previous work suggests that social psychological experiments typically result in a small to medium effect size (around .3; Richard, Bond, and Stokes-Zoota, 2003). Given that effect size, cell sizes of 45 (which is well exceeded) would provide an $80 \%$ chance of detecting the effect.

Finally, building off of the work by Leahey and colleagues (2011), a social network analysis is needed to assess spread of WSC in peer groups and how conversations about norms for weight may influence the spread and severity of WSC. Given that WSC has demonstrated negative psychological and physical outcomes (Hunger and Major, 2015), designing interventions in which individuals could affirm their identities before experiencing weight based stigmatization could help buffer these negative effects. Additionally, teaching people to shift the focus of their conversations away from weight and towards health could result in more positive outcomes for the overweight and obese (Hunger and Tomiyama, 2015). Findings from these studies are the first step in establishing regional differences in WSC and also in highlighting the relationship between WSC, BMI, and peer groups across the US.

\section{References}

Albuquerque, D., Stice, E., Rodríguez-López, R., Manco, L., and Nóbrega, C. (2015). Current review of genetics of human obesity: from molecular mechanisms to an evolutionary perspective. Molecular Genetics and Genomics, 290, 1191-1221.

Bennett, G.G., and Wolin, K.Y. (2006). Satisfied or unaware? Racial differences in perceived weight status. International Journal of Behavioral Nutrition and Physical Activity, 3, 40.

Burke, M.A., Heiland, F.W., and Nadler, C. M. (2010). From "overweight" to "about right": evidence of a generational shift in body weight norms. Obesity, 18, 1226-1234.

Centers for Disease Control (2014). About BMI for Adults. Retrieved from http://www.cdc.gov/healthyweight/ assessing/bmi/adult_ bmi/index.html

Centers for Disease Control. (2015). [County level obesity rate information]. Community Health Status Indicators. Retrieved from http://wwwn.cdc.gov/CommunityHealth/homepage.aspx

Christakis, N.A., and Fowler, J.H. (2007). The spread of obesity in a large social network over 32 years. New England Journal of Medicine, 357, 370-379. 
Christakis, N.A., and Fowler, J.H. (2008). The collective dynamics of smoking in a large social network. New England Journal of Medicine, 358, 2249-2258.

Dwyer-Lindgren, L., Freedman, G., Engell, R., Fleming, T., Lim, S., Murray, C., and Mokdad, A. (2013). Prevalence of physical activity and obesity in US counties, 2001-2011: A road map for action. Population Health Metrics, 11, 1- 11.

Fikkan, J.L., and Rothblum, E.D. (2012). Is fat a feminist issue? Exploring the gendered nature of weight bias. Sex Roles, 66, 575592.

Finkelstein, E., Khavjou, O., Thompson, H., Trogdon, J., Pan, L., Sherry, B., and Dietz, W. (2012). Obesity and severe obesity forecasts through 2030. American Journal of Preventive Medicine, 42, 563570 .

Hatzenbuehler, M.L., Keyes, K.M., and Hasin, D.S. (2009). Associations between perceived weight discrimination and the prevalence of psychiatric disorders in the general population Obesity, 17, 20332039.

Hayes, A.F. (2012). PROCESS: A versatile computational tool for observed variable mediation, moderation, and conditional process modeling [White paper]. Retrieved from http://www.afhayes.com/public/process2012.pdf

Hayes, A.F. (2015). An index and test of linear moderated mediation. Multivariate Behavioral Research, 50, 1-22.

Hunger, J.M., and Major, B. (2015). Weight Stigma Mediates the Association Between BMI and Self-Reported Health. Health Psychology, 34, 172-175.

Hunger, J.M., Major, B., Blodorn, A., and Miller, C. (2015). Weighed down by stigma: How weight-based social identity threat influences weight gain and health. Social Psychology and Personality Compass, 9, 255-268.

Hunger, J.M., and Tomiyama, A.J. (2015). A Call to Shift the Public Health Focus Away From Weight. American Journal of Public Health, 105, e3.

Latner, J.D., Stunkard, A.J., and Wilson, G.T. (2005). Stigmatized students: Age, sex, and ethnicity effects in the stigmatization of obesity. Obesity Research, 13, 1226-1231.

Lavie, C.J., McAuley, P.A., Church, T.S., Milani, R.V., and Blair, S.N. (2014). Obesity and cardiovascular diseases: Implications regarding fitness, fatness, and severity in the obesity paradox. Journal of the American College of Cardiology, 63, 1345-1354.

Leahey, T.M., Kumar, R., Weinberg, B.M., and Wing, R.R. (2012). Teammates and social influence affect weight loss outcomes in a team based weight loss competition. Obesity, 20, 1413-1418.

Leahey, T.M., LaRose, J.G., Fava, J.L., and Wing, R.R. (2011). Social influences are associated with BMI and weight loss intentions in young adults. Obesity, 19, 1157-1162.

Major, B., Eliezer, D., and Rieck, H. (2012). The psychological weight of weight stigma. Social Psychological and Personality Science, 3, 651658.

Major, B., Hunger, J.M., Bunyan, D.P., and Miller, C.T. (2014). The ironic effects of weight stigma. Journal of Experimental Social Psychology, 51, 74-80.

Muennig, P., Jia, H., Lee, R., and Lubetkin, E. (2008). I think therefore I am: Perceived ideal weight as a determinant of health. American Journal of Public Health, 98, 501-506.

Ogden, C.L., Carroll, M.D., Kit, B.K., and Flegal, K.M. (2014). Prevalence of childhood and adult obesity in the United States, 2011-2012. Journal of the American Medical Association, 311, 806-814.

Pearl, R.L., and Puhl, R.M. (2016). The distinct effects of internalizing weight bias: An experimental study. Body Image, 17, 38-42.

Piko, B.F., Luszczynska, A., Gibbons, F.X., and Teközel, M. (2005). A culture-based study of personal and social influences of adolescent smoking. The European Journal of Public Health, 15, 393-398.

Puhl, R., and Heuer, C. (2010). Obesity Stigma: Considerations for Public Health. American Journal of Public Health, 100, 1019-1028.

Rand, C.S., and Kuldau, J.M. (1990). The epidemiology of obesity and self defined weight problem in the general population: Gender, race, age, and social class. International Journal of Eating Disorders, 9, 329-343.

Richard, F.D., Bond Jr, C.F., and Stokes-Zoota, J.J. (2003). One hundred years of social psychology quantitatively described. Review of General Psychology, 7, 331-363.

Robinson, E., Thomas, J., Aveyard, P., and Higgs, S. (2014). What everyone else is eating: A systematic review and meta-analysis of the effect of informational eating norms on eating behavior. Journal of the Academy of Nutrition and Dietetics, 114, 414-429.

Rosenquist, J.N., Murabito, J., Fowler, J.H., and Christakis, N.A. (2010). The spread of alcohol consumption behavior in a large social network. Annals of Internal Medicine, 152, 426-433.

Rothblum, E.D. (1992). The stigma of women's weight: Social and economic realities. Feminism and Psychology, 2, 61-73.

Schutz, H.K., Paxton, S.J., Wertheim, E.H. (2002). Investigation of body comparison among adolescent girls1. Journal of Applied Social Psychology, 32, 1906-1937.

Sobal, J. (2004). Sociological analysis of the stigmatisation of obesity. In J. Germov and L. Williams (Eds.), A Sociology of Food and Nutrition: The Social Appetite ( $2^{\text {nd }}$ ed.). Melbourne, Australia: Oxford University Press.

The State of Obesity. (2014). [Graph illustration of obesity rates by state]. Adult Obesity in the United States. Retrieved from http://stateofobesity.org/adult-obesity/

Tomiyama, A.J. (2014). Weight stigma is stressful. A review of evidence for the cyclic obesity/weight-based stigma model. Appetite, 82, 8-15.

Ulijaszek, S.J. (2012). Socio-economic status, forms of capital and obesity. Journal of Gastrointestinal Cancer, 43, 3-7.

Vartanian, L.R., Novak, S.A. (2011). Internalized societal attitudes moderate the impact of weight stigma on avoidance of exercise. Obesity, 19, 757-762.

Wells, J.C.K. (2012). Ethnic variability in adiposity, thrifty phenotypes and cardiometabolic risk: Addressing the full range of ethnicity, including those of mixed ethnicity. Obesity Reviews, 13, 14-29. 